\title{
The Dipole Phase in the Two-Dimensional Hierarchical Coulomb Gas: Analyticity and Correlations Decay
}

\author{
G. Benfatto ${ }^{\star}$, G. Gallavotti ${ }^{\star \star}$, and F. Nicolò ${ }^{\star \star \star}$ \\ Mathematics Department, Rutgers University, Hill Center, New Brunswick, NJ 08903, USA \\ Dipartimento di Matematica, Il Università di Roma, Via O. Raimondo, I-00173 Roma, Italy
}

\begin{abstract}
We illustrate the mechanism producing the dipole phase in a two dimensional Coulomb system by a detailed analysis of a hierarchical model. We prove the analyticity of the pressure and of the correlations for $\alpha^{2} \equiv \beta e^{2}>8 \pi$ (i.e. right above the usually conjectured value for the KosterlitzThouless phase transition). We find also a power law decay for the correlations with exponent $\alpha^{2} / 2 \pi$ as the hierarchical distance goes to infinity.
\end{abstract}

\section{Introduction}

The renormalization group theory of the ultraviolet stability for scalar fields [1-3] hinted that the same techniques could be employed in the analysis of the two dimensional Coulomb gas of identical charges $\pm e$ even, and particularly, in the regime of the Kosterlitz-Thouless phase. The latter was rigorously established [4] for low temperatures, i.e. for $\beta e^{2} \equiv \alpha^{2} \gg 8 \pi, \beta$ being the inverse temperature and the Coulomb potential being normalized as

$$
V(x-y) \simeq \frac{1}{2 \pi} \log |x-y|^{-1} \quad \text { as } \quad|x-y| \rightarrow \infty
$$

and regularized at short distance.

This paper is a continuation of the program started in [3] and continued in [5-7] to study the problem of the molecules formation in the two dimensional Coulomb gas and the related structure of the transition from the plasma phase to the dipole phase (Kosterlitz-Thouless transition).

\footnotetext{
* Partially supported by "Ministero della Pubblica Istruzione", Gruppo Nazionale per la Fisica Matematica del CNR" and Grant A.F.O.S.R.-82-0016C

$\star \star$ Partially supported by "Ministero della Pubblica Istruzione" and Grant N.S.F. DMS 8503333

$\star \star \star$ Partially supported by "Ministera della Pubblica Istruzione"
} 
The arguments leading to the Kosterlitz-Thouless phase transition establish that the transition point is $\alpha^{2}=8 \pi$, in the zero density limit. The new phase appears to have various properties, among which

1) power law decay of the correlations,

2) the free energy and all the other thermodynamical quantities are analytic in the density at $\varrho=0$.

In [4] it was shown that the correlations decay as a power law for $\alpha^{2} \gg 8 \pi$. Here we show, in a hierarchical model, that this holds for $\alpha^{2}>8 \pi$ and at the same time we show the analyticity of the pressure at zero density.

In $[1-3,5-7]$ it has been abundantly shown that the understanding of the hierarchical model is a preliminary step to the solution of many field theory or statistical mechanics problems: the "missing details" to understand the real physical problem are usually rather lengthy but trivial and not too interesting. In this case we are not perhaps in the same situation, but we hope that our techniques yield at least a key to the understanding of the ordinary two dimensional Coulomb gas with ultraviolet regularization, even if it turns out to have different analyticity properties in the activity.

From a technical viewpoint this paper relies on the new proof in [8] of the convergence of the Mayer expansion for the Yukawa gas.

\section{The Model}

We suppose the reader familiar with the sine-Gordon representation of the two dimensional Coulomb gas used in [5]: see [10, Sects. 11 and 15] for a review of the formalism.

Let $\Lambda$ be a finite box enclosing the system: then we consider the grand canonical partition function $Z$ for a neutral system of charges $\sigma e, \sigma= \pm 1$, with activity $\frac{\lambda}{2}$ and temperature $\beta^{-1}$ such that $\beta e^{2}=\alpha^{2}$, interacting with a potential

$$
\begin{gathered}
V_{x y}=C_{x y}-C_{00}, \\
C_{x y}=\frac{1}{(2 \pi)^{2}} \int \frac{C(p)}{p^{2}+g^{-2 R}} e^{i p(x-y)} d^{2} p,
\end{gathered}
$$

where $g>1$ and $g^{R}$ is an infrared regularization, while $C(0)=1$ and $C(p) \rightarrow 0$, as $p \rightarrow \infty$, fast enough to act as an ultraviolet regularization (necessary because otherwise the Coulomb system would be unstable for $\alpha^{2} \geqq 4 \pi$ ).

The Coulomb gas partition function $Z$ can then be written as

$$
Z(\Lambda, \beta, \lambda)=\lim _{R \rightarrow \infty} \int P\left(d \phi^{(<R)}\right) \exp \left(\sum_{\sigma= \pm 1} \frac{\lambda}{2} g^{2 R} e^{-\frac{\alpha^{2}}{2} C_{\mathrm{o} o}^{(<R)}} \int_{\Lambda g^{-R}}: e^{i \sigma \alpha \phi_{\xi}^{(<R)}}: d \xi\right),
$$

where $\phi_{\xi}^{(<R)}$ is a gaussian random field with covariance

$$
C_{\xi \eta}^{(<R)}=\mathscr{E}\left(\phi_{\xi}^{(<R)} \phi_{\eta}^{(<R)}\right)=\frac{1}{(2 \pi)^{2}} \int_{R^{2}} e^{i p(\xi-\eta)} \frac{C\left(p g^{-R}\right)}{p^{2}+1} d^{2} p .
$$


For a derivation of the (2.2), (2.3) see [5] (or [10, Sect. 15, (15.19)] with $N=-1$, i.e. no $\psi$-field).

One gets a possible model by choosing, see $[5,10], C(p)=\frac{1-g^{-2 R}}{p^{2}+1}$ so that

$$
\frac{C\left(p g^{-R}\right)}{p^{2}+1}=\sum_{j=0}^{R-1}\left(\frac{1}{p^{2}+g^{2 j}}-\frac{1}{p^{2}+g^{2(j+1)}}\right)=\sum_{j=0}^{R-1} \bar{C}\left(g^{-j} p\right) g^{-2 j}
$$

This is a useful decomposition because it shows that $\phi^{(<R)}$ in (2.2) can be realized as

$$
\phi_{x}^{(<R)}=\sum_{j=0}^{R-1} \phi_{x}^{(j)}
$$

where $\phi_{x}^{(j)}$ are independent gaussian fields "living on scale $g^{-j "}: \phi_{x}^{(j)}$ has the same distribution as $\phi_{g^{J} x}^{(0)}$. Moreover, by direct calculation

$$
C_{00}=\frac{R}{2 \pi} \log g .
$$

The hierarchical model replaces (2.5) by a sum of the same type but with new fields $\phi_{x}^{(j)}$ which are defined to be

$$
\phi_{x}^{(j)} \equiv z_{\Delta} \text { if } \quad x \in \Delta,
$$

where $\Delta$ is a square of side size $g^{-j}$ extracted from a pavement $Q_{j}$ of $R^{2}$ with squares of size $g^{-j}, g$ is an integer and $Q_{j+1}$ is obtained from $Q_{j}$ dividing each tessera $\Delta \in Q_{j}$ in $g^{2}$ tesserae.

The $z_{\Delta}$ are gaussian random variables independently distributed and with covariance

$$
\mathscr{E}\left(z_{\Delta}^{2}\right)=\frac{1}{2 \pi} \log g
$$

The hierarchical Coulomb gas is by definition given by $(2.2)$ with $\phi^{(<R)}$ given by (2.5) and $\phi^{(j)}$ given by (2.7). This amounts to replacing the potential $V_{x y}$ between the charges into the function given by (2.1) with $C_{x y}$ given by

$$
C_{x y}=\frac{1}{2 \pi} \log g \sum_{\substack{\Delta: \\ \Delta \ni g^{-R_{x}, g^{-R} y}}} 1=\frac{1}{2 \pi} \log d^{h}\left(g^{-R} x, g^{-R} y\right),
$$

where $d^{h}(u, v)$ is the "hierarchical" distance between $u$ and $v$, i.e. $d^{h}(u, v)=g^{-h(u, v)}$ with $h(u, v)$ being the largest integer $h$ such that there is a $\Delta \in Q_{h}$ containing both $u$ and $v$. One can also define in a natural way a "hierarchical" distance $\widetilde{d}(x, y)$ between two points $x, y$ belonging to the original physical space, by the formula

$$
\tilde{d}(x, y)=g^{R-h\left(g^{-R} x, g^{-R} y\right)} .
$$

Then one can write the "hierarchical" Coulomb potential in the following form

$$
V_{x y}=-\frac{1}{2 \pi} \log \tilde{d}(x, y) .
$$


The potential (2.11) differs from (2.1) because it is not translation invariant in a rather substantial way. Nevertheless one expects that it produces a model rather similar in many respects to the true Coulomb gas: in particular one expects for this model the kind of phase transitions of molecule formations which lead from a plasma phase at small $\alpha^{2}$ to a dipole phase at $\alpha^{2} \gg 8 \pi$.

The correlation functions can be easily expressed as functional integrals. For instance the $n$-charges truncated correlation functions are

$$
\begin{aligned}
\varrho_{\Lambda}^{T}\left(x_{1}, \sigma_{1}, \ldots, x_{n}, \sigma_{n}\right)= & \frac{\partial^{n}}{\partial \omega_{1} \ldots \partial \omega_{n}} \log \int P\left(d \phi^{(<R)}\right) \\
& \times \exp \left[\left(\sum_{\sigma= \pm 1} \frac{\lambda}{2} g^{\left(2-\frac{\alpha^{2}}{4 \pi}\right) R} \int_{\Lambda g^{-R}}: e^{i \sigma \alpha \phi_{x}^{(<R)}}: d x\right)\right. \\
& \left.+\left(\frac{\lambda}{2} \sum_{j=1}^{n} \omega_{j} g^{-\frac{\alpha^{2}}{4 \pi} R}: e^{\left.i \sigma \alpha \phi_{g}^{(<R)}\right)}\right)\right]\left.\right|_{\underline{\omega}=\underline{0}} .
\end{aligned}
$$

The (2.2), (2.12) show that the Coulomb gas problem can be interpreted as an ultraviolet stability problem with an ultraviolet cutoff at scale $g^{-R}$ if $g^{R}$ is the infrared cutoff in the original problem. Since the model is rather simple it can be studied rather well with the methods of field theory.

\section{Recursion Relation for the Coefficients of the Mayer Expansion}

The integral over $\phi^{(<R)}$ can be performed by using the renormalization group technique of integrating successively out the high frequency components of $\phi^{(<R)}$, i.e. $\phi^{(<R-1)}, \phi^{(<R-2)}, \phi^{(<R-3)} \ldots$.

It is well known that this is a very efficient algorithm [11].

Calling $V^{(<R)}$ the function of $\phi^{(<R)}$ in the exponential in (2.2), we define $V^{(\leqq k)}$ for $k=0,1, \ldots, R-1$ recursively as

$$
e^{V(\leqq k)}=\int P\left(d \phi^{(k+1)}\right) e^{V(\leqq k+1)}
$$

where the function $e^{V(\leqq k)}$ is a function of

$$
\phi^{(\leqq k)}=\sum_{j=0}^{k} \phi^{(j)} \text {. }
$$

The function $V^{(\leqq k)}$ is easily expanded in terms of a formal series:

$$
V^{(\leqq k)}=\sum_{t=1}^{\infty} \frac{1}{t !} \mathscr{E}_{k+1}^{T} \underbrace{\left(V^{(\leqq k+1)}, \ldots, V^{(\leqq k+1)}\right)}_{t},
$$

where $\mathscr{E}_{k+1}$ denotes the integration with respect to the field $\phi^{(k+1)}$ and $\mathscr{E}_{k+1}^{T} \underbrace{\left(V^{(\leqq k+1)}, \ldots, V^{(\leqq k+1)}\right)}_{t}=\left.\frac{\partial^{t}}{\partial \omega_{1} \ldots \partial \omega_{t}} \log \mathscr{E}_{k+1}\left(\exp \left(\omega_{1}+\ldots+\omega_{t}\right) V^{(\leqq k+1)}\right)\right|_{\underline{\omega}=\underline{0}}$. 
Fig. 1

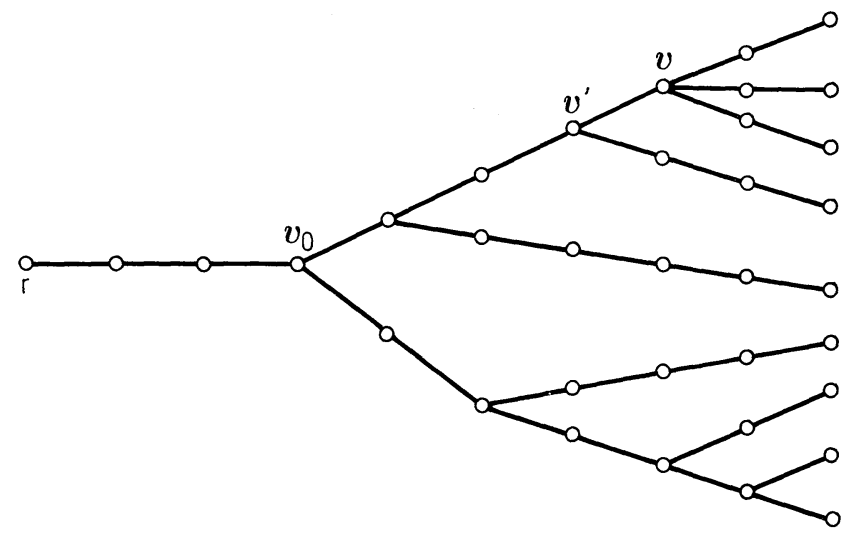

From (3.3) it is easy to deduce an expression for $V^{(\leqq k)}$ by just iterating it, see [5, (6.10)]. As the reader can check immediately, the iteration of (3.3) leads to an expression of $V^{(\leqq k)}$ in terms of trees:

$$
V^{(\leqq k)}=\sum_{\gamma} \frac{V(\gamma)}{n(\gamma)},
$$

where $\gamma$ is a tree with $n$ endpoints $1,2, \ldots, n$ and some (possibly none) non-trivial vertices $v, w$ and one "root" $r$.

\section{Moreover}

1) each vertex $v$ carries a "frequency index" $h_{v}=-1,0,1,2, \ldots$, so that $h_{r}=k$ and $h_{v}$ grows by one unit at a time as one climbs the tree from $r$ upwards until it reaches the value $R-1$ at the last inner vertices.

2) each vertex $v$, except the root $r$, with frequency index $h_{v}$ and out of which emerge $s_{v}$ branches, represents a truncated expectation $\mathscr{E}_{h_{v}}^{T}$ of order $s_{v}$ : if the vertex $v$ is trivial, i.e. no true bifurcation takes place at $v$, then it symbolically represents an ordinary expectation.

3) the factor $n(\gamma)$ is

$$
n(\gamma)=\prod_{v} s_{v} !
$$

4) the endpoints represent the function

$$
\sum_{\sigma= \pm 1} \frac{\lambda}{2} g^{\left(2-\frac{\alpha^{2}}{4 \pi}\right) R} \int_{\Lambda g^{-R}}: e^{i \sigma \alpha \phi_{x}^{(<R)}}: d x .
$$

Therefore $V(\gamma)$ has a rather complicated expression. However, since

$$
: e^{i \sigma \alpha \phi_{x}^{(<R)}}:=e^{+\frac{\alpha^{2}}{2} \mathscr{E}\left(\phi_{x}^{(<R)}\right)} e^{i \sigma \alpha \phi_{x}^{(<R)},}
$$

it follows easily (see [5], or [10, Sects. 11 and 15] for details) that $V(\gamma)$ must have the form of an integral of a kernel $\bar{V}(\gamma, \underline{\sigma}, \underline{x})$ depending on $\gamma$, on $\underline{\sigma}=\left(\sigma_{1}, \ldots, \sigma_{n}\right)$, $\sigma_{i}= \pm 1$, and on $\underline{x}=\left(x_{1}, \ldots, x_{n}\right) \in \mathbb{R}^{2 n}, n \equiv|\gamma|$ being the number of endpoints of $\gamma$, times : $\exp i \alpha \phi^{(\leqq k)}(\underline{x}, \underline{\sigma})$ :, where

$$
\phi^{(\leqq k)}(\underline{x}, \underline{\sigma})=\sum_{i=1}^{n} \phi_{x_{i}}^{(\leqq k)} \sigma_{i},
$$


i.e.

$$
V(\gamma)=\sum_{\underline{\sigma}\left(\gamma^{-R} \Lambda\right)^{n}} d x_{1} \ldots d x_{n} \bar{V}(\gamma, \underline{\sigma}, \underline{x}): \exp i \alpha \phi^{(\leqq k)}(\underline{x}, \underline{\sigma}): .
$$

To discuss the structure of $\bar{V}(\gamma, \underline{\sigma}, \underline{x})$ it will be convenient to think $(\gamma, \underline{\sigma}, \underline{x})$ as a tree $\gamma$ with labels $x_{1}, \sigma_{1}$ attached to the first endpoint, $x_{2}, \sigma_{2}$ to the second, etc.

In this way it will be natural to think that each non-trivial vertex $v$ of $\gamma$ represents a cluster $\underline{x}(v)$ of charges $\underline{\sigma}(v)$ : such cluster consists of the point charges $\left(x_{i}, \sigma_{i}\right)$ which label the endpoints $i \in v$, i.e. the endpoints of $\gamma$ which can be reached by climbing the tree from $v$ upwards. The charge of the cluster associated with $v \in \gamma$ is defined as

$$
Q_{v}=\sum_{i \in v} \sigma_{i}
$$

With the above notations and using the properties of gaussian integrals and the elementary relations

$$
\begin{aligned}
& : e^{i \alpha \phi^{(\leqq k)}(\underline{x}, \underline{\sigma})}:=: e^{i \alpha \phi^{(<k)}(\underline{x}, \underline{\sigma})}:: e^{i \alpha \phi^{(k)}(\underline{x}, \underline{\sigma})}: \\
& : e^{i \alpha \phi^{(<k)}(\underline{x}, \underline{\sigma})}:=e^{+\frac{\alpha^{2}}{2} U^{(<k)}(\underline{x}, \underline{\sigma})} e^{i \alpha \phi^{(<k)}(\underline{x}, \underline{\sigma})},
\end{aligned}
$$

where

$$
U^{(<k)}(\underline{x}, \underline{\sigma})=\sum_{i=1}^{n} \sum_{j=1}^{n} \sigma_{i} \sigma_{j} C_{x_{i} x_{j}}^{(<k)}
$$

one realizes by induction (see $[5,(6.5)]$ or $[10$, Sect. $11,(11.4)-(11.7)$ and Sect. 15], for more details) that, if $\gamma$ has at least one non-trivial vertex and if $v$ is the first nontrivial vertex of $\gamma$, with frequency label $k$, followed by $s$ vertices $v_{1}, v_{2}, \ldots, v_{s}$ (either non-trivial or endpoints), then

$$
\begin{aligned}
\bar{V}(\gamma, \underline{\sigma}, \underline{x})= & \left(\prod_{i=1}^{s} \bar{V}\left(\gamma_{i}, \underline{\sigma}_{i}, \underline{x}_{i}\right)\right) e^{-\frac{\alpha^{2}}{2}\left[U^{(<k)}(\underline{x}, \underline{\sigma})-\sum_{i=1}^{s} U(<k)\left(\underline{x}_{i}, \underline{\sigma}_{i}\right)\right]} \\
& \times \mathscr{E}_{k}^{T}\left(: e^{i \alpha \phi^{(k)}\left(\underline{x}_{1}, \underline{\sigma}_{1}\right)}:, \ldots,: e^{i \alpha \phi^{(k)}\left(\underline{x}_{s}, \underline{\sigma}_{s}\right)}:\right),
\end{aligned}
$$

where $\gamma_{1}, \ldots, \gamma_{s}$ are the $s$ subtrees growing out of the vertex $v$ thought of as a new root and $\underline{\sigma}_{i} \equiv \underline{\sigma}\left(v_{i}\right), \underline{x}_{i} \equiv \underline{x}\left(v_{i}\right)$.

If $\gamma$ has no non-trivial vertex, it is:

$$
\bar{V}(\gamma, \sigma, x)=\frac{\lambda}{2} g^{\left(2-\frac{\alpha^{2}}{4 \pi}\right) R} .
$$

Clearly (3.13), (3.14) allow us to find an explicit formula for $\bar{V}$ (see [5] or [10, (11.5)-(11.7) and (15.9)-(15.22)]); if $h_{r}=-1$ :

$$
\begin{aligned}
\bar{V}(\gamma, \underline{\sigma}, \underline{x})= & \prod_{v \text { non-trivial }}\left\{e^{-\frac{\alpha^{2}}{2}\left[U\left(<h_{v}\right)\left(\underline{x}_{v}, \underline{\sigma}_{v}\right)-\sum_{i-1}^{s_{v}} U\left(<h_{v}\right)\left(\underline{x}_{v_{v}}, \sigma_{v_{i}}\right)\right.} F(v)\right\}\left[\frac{\lambda}{2} g^{\left(2-\frac{\alpha^{2}}{4 \pi}\right) R}\right]^{n} \\
= & \prod_{v \text { non-trivial }}\left\{e^{-\frac{\alpha^{2}}{2}\left[U^{\left.\left(<h_{v}\right)\left(\underline{x}_{v}, \underline{\sigma} \underline{\sigma}_{v}\right)-U\left(<h_{v}\right)\left(\underline{x}_{v}, \underline{\sigma}_{v}\right)\right]} F(v)\right\}}\right. \\
& \times \prod_{i=1}^{n}\left(g^{\frac{\alpha^{2}}{4 \pi} h_{i}} \frac{\lambda}{2} g^{\left(2-\frac{\alpha^{2}}{4 \pi}\right) R}\right)
\end{aligned}
$$


where the last product is over the endpoints $i$ of $\gamma, v^{\prime}$ is either the non-trivial vertex immediately preceding $v$ or the root when such a vertex does not exist, $h_{i}$ is the frequency of the non-trivial vertex to which the $i$-th endpoint is attached, $F(v)$ is a shorthand symbol for the truncated expectation in (3.13) and we used the definitions: $U^{(<0)} \equiv 0 \equiv C^{(<0)} \equiv \phi^{(<0)}$.

The key problem remaining before us is to find what to do with the truncated expectations in $F(v)$ : we have two convenient representations of $F(v)$. Namely (see [10, Eq. (C23)]):

$$
\begin{aligned}
F(v)= & \sum_{\tau \in \mathscr{G}_{v}^{c}} \prod_{\lambda \in \tau}\left[e^{-\alpha^{2} C^{\left(h_{v}\right)}\left(\underline{x}_{v^{\prime}}, \underline{\sigma}_{v^{\prime}} ; \underline{x}_{v^{\prime \prime}}, \underline{\sigma}_{v^{\prime \prime}}\right)}-1\right]=\frac{1}{(2 \pi i)^{s_{v}}} \oint \prod_{j=1}^{s_{v}} \frac{d \zeta_{j}}{\zeta_{j}^{2}} \\
& \times \log \left[\sum_{I \cong\left\{1, \ldots, s_{v}\right\}}\left(\prod_{i \in I} \zeta_{i}\right) \exp -\alpha^{2} \sum_{i<j ; i, j \in I} C^{\left(h_{v}\right)}\left(\underline{x}_{v_{i}}, \underline{\sigma}_{v_{i}} ; \underline{x}_{v_{j}}, \underline{\sigma}_{v_{j}}\right)\right]
\end{aligned}
$$

where $\lambda$ denotes a pair $v^{\prime}, v^{\prime \prime} \in\left\{v_{1}, \ldots, v_{s_{v}}\right\}$ of vertices among the $s_{v}$ that follow immediately $v, \mathscr{G}_{v}^{c}$ denotes the family of all connected Mayer graphs with vertices $\left\{v_{1}, \ldots, v_{s_{v}}\right\} \quad$ and, $\quad$ if $\quad \underline{x}=\left(x_{1}, \ldots, x_{p}\right), \quad \underline{\sigma}=\left(\sigma_{1}, \ldots, \sigma_{p}\right), \quad \underline{x}^{\prime}=\left(x_{p+1}, \ldots, x_{q}\right)$, $\underline{\sigma}^{\prime}=\left(\sigma_{p+1}, \ldots, \sigma_{q}\right)$ :

$$
C^{(h)}\left(\underline{x}, \underline{\sigma} ; \underline{x}^{\prime}, \underline{\sigma}^{\prime}\right)=\sum_{i=1}^{p} \sum_{j=p+1}^{q} \sigma_{i} \sigma_{j} C_{x_{i} x_{j}}^{(h)} .
$$

This is the mutual electrostatic energy of the clusters $(\underline{x}, \underline{\sigma})$ and $\left(\underline{x}^{\prime}, \underline{\sigma}\right)$ : notice that

$$
\sum_{i<j, i, j \in I} C^{\left(h_{v}\right)}\left(\underline{x}_{v_{i}}, \underline{\sigma}_{v_{i}} ; \underline{x}_{v_{j}}, \underline{\sigma}_{v_{j}}\right)=U^{(h)}\left(\bigcup_{i \in I} \underline{x}_{v_{i}}, \bigcup_{i \in I} \underline{\sigma}_{v_{i}}\right)-\sum_{i \in I} U^{(h)}\left(\underline{x}_{v_{i}}, \underline{\sigma}_{v_{i}}\right),
$$

where $U^{(h)}(\underline{x}, \sigma)$ is the electrostatic energy of the charges $(\underline{x}, \underline{\sigma})$ with the potential $C^{(h)}$, see (3.12).

So far we did not use the structure of the hierarchical model, i.e. the special form of $C^{(h)}$ :

$$
C_{x y}^{(h)}= \begin{cases}0, & \text { if } x, y \text { are not in the same } \Delta \in Q_{h} \\ \frac{\log g}{2 \pi} & \text { otherwise }\end{cases}
$$

In this case the following simplifications occur:

1) $F(v)=0$ unless all points in the cluster $\underline{x}(v)$ are inside the same box $\Delta$ of size $g^{-h_{v}}$,

2) $F(v)=0$ unless all the charges of the largest subclusters $v_{1}, \ldots, v_{s_{v}}$ in $v$, i.e. the subclusters associated with the vertices immediately following $\gamma$, are non-zero: $Q_{v_{i}} \neq 0, i=1, \ldots, s_{v}$

$$
\begin{gathered}
U^{\left(<h_{v}\right)}(\underline{x}(v), \underline{\sigma}(v))=\left(\sum_{i \in v} \sigma_{i}(v)\right)^{2} \frac{\log g}{2 \pi} h_{v}=Q_{v}^{2} \frac{\log g}{2 \pi} h_{v} \\
C^{\left(h_{v}\right)}\left(\underline{x}_{v_{i}}, \underline{\sigma}_{v_{i}} ; \underline{x}_{v_{j}}, \underline{\sigma}_{v_{j}}\right)=\frac{\log g}{2 \pi} Q_{v_{i}} Q_{v_{j}}
\end{gathered}
$$

Furthermore we shall use:

$$
\begin{aligned}
\sum_{\text {endpoints } i} C_{00}^{\left(<h_{i}\right)=}= & \frac{\log g}{2 \pi} \sum_{i} h_{i}=\frac{\log g}{2 \pi}\left(\sum_{v \text { n.t. }} n_{v}\left(h_{v}-h_{v^{\prime}}\right)+n h_{r}\right), \\
& \sum_{v^{\prime} \geqq v \text { n.t. }}\left(s_{v^{\prime}}-1\right)=n_{v}-1,
\end{aligned}
$$


where $n_{v}$ is the number of endpoints that can be reached by climbing $\gamma$ from $v$, i.e. is the number of charges in the cluster $x(v)$; here n.t. means "non trivial."

Collecting the above four properties, (3.20), (3.15), and calling $v_{0}$ the first nontrivial vertex of $\gamma$ we find

$$
\begin{aligned}
\frac{1}{|\Lambda|} \log Z= & \beta p(\beta, \lambda)=\frac{1}{|\Lambda|} \sum_{g, \gamma, h_{r}=-1}^{*} \int_{\Lambda g^{-R}} \frac{d x_{1} \ldots d x_{n}}{\prod_{v} s_{v} !} \prod_{v} F(v) \\
& \times \prod_{v \text { n.t. }} g^{-\frac{\alpha^{2}}{4 \pi} Q_{v}^{2}\left(h_{v}-h_{v^{\prime}}\right)+\frac{\alpha^{2}}{4 \pi} n_{v}\left(h_{v}-h_{v^{\prime}}\right)}\left(\frac{\lambda}{2} g^{\left(2-\frac{\alpha^{2}}{4 \pi}\right) R} g^{-\frac{\alpha^{2}}{4 \pi}}\right)^{n},
\end{aligned}
$$

where $\Sigma^{*}$ means that the sum is restricted to the charge configurations $\sigma$ such that $Q_{v} \neq 0$ if $v>v_{0}$. Equation (3.22) is the Mayer series for our Coulomb system.

The property 1) of $F(v)$ strongly restricts the variability of $x_{1}, \ldots, x_{n}$ forcing $x(v)$ to be made of particles all in the same box of size $g^{-h_{v}}$ : this allows us to calculate the integrals.

Let $F^{*}(v)$ be the value of $F(v)$ when $x(v)$ are all inside the same box $\Delta$ of size $g^{-h_{v}}$ [see (3.16) and the property 4)]:

$$
F^{*}(v)=\sum_{r} \prod_{\lambda=\left(v_{i}, v_{j}\right)}\left(g^{-\frac{\alpha^{2}}{4 \pi} Q_{v_{i}} Q_{v_{j}}}-1\right) .
$$

Then, using the mentioned property 1), one finds

$$
\lim _{\Lambda \rightarrow \infty} \frac{1}{|\Lambda|} \int_{\Lambda g^{-R}} d x_{1} \ldots d x_{n} \prod_{v} F(v)=g^{-2 R} \prod_{v \text { n.t. }} F^{*}(v) g^{-2\left(s_{v}-1\right) h_{v}},
$$

where the convergence in (3.24) is dominated in the sense that the right-hand side is always larger or equal to the left-hand side.

The (3.24) gives with (3.22) the following bound on the $n$-th order coefficient $\mu_{n,}, n>1$, of the Mayer expansion: if $a=\frac{\alpha^{2}}{4 \pi}-2$,

$$
\begin{aligned}
\left|\mu_{n}\right| \leqq & g^{-2 R+2(n-1)}\left(\frac{1}{2} g^{-\frac{\alpha^{2}}{4 \pi}-a R}\right)^{n} \\
& \times \sum_{\gamma, h_{r}=-1}^{*} \prod_{v \text { n.t. }}\left[g^{-\frac{\alpha^{2}}{4 \pi} Q_{v}^{2}\left(h_{v}-h_{v^{\prime}}\right)+\frac{\alpha^{2}}{4 \pi} n_{v}\left(h_{v}-h_{v^{\prime}}\right)} g^{-2\left(n_{v}-1\right)\left(h_{v}-h_{v^{\prime}}\right)} \frac{\left|F^{*}(v)\right|}{S_{v} !}\right]
\end{aligned}
$$

having used the equality immediately following from (3.21) (recall our convention $\left.h_{r}=-1\right)$

$$
\sum_{v}\left(s_{v}-1\right) h_{v}=\sum_{v}\left(n_{v}-1\right)\left(h_{v}-h_{v^{\prime}}\right)-(n-1) .
$$

To estimate $F^{*}(v)$ we use the second of (3.16) and properties 3), 4):

$$
F^{*}(v)=\frac{1}{(2 \pi i)^{s_{v}}} \oint\left(\prod_{i=1}^{s_{v}} \frac{d \zeta_{i}}{\zeta_{i}^{2}}\right) \log \sum_{I \subset\left\{1, \ldots, s_{v}\right\}}\left(\prod_{i \in I} \zeta_{i}\right) g^{-\frac{\alpha^{2}}{4 \pi}\left(Q_{v_{I}}^{2}-\sum_{i \in I} Q_{v_{i}}^{2}\right)}
$$

where we used the shorthand $Q_{v_{I}}=\sum_{i \in I} Q_{v_{i}}$. 
Therefore, by changing $\zeta_{i} \rightarrow \zeta_{i} g^{\frac{\alpha^{2}}{4 \pi} Q^{2}} \equiv \zeta_{i}^{\prime}$, we find

$$
F^{*}(v)=g^{\frac{\alpha^{2}}{4 \pi} \sum_{v_{i}} Q_{v_{j}}^{2}} \frac{1}{(2 \pi i)^{s_{v}}} \oint\left(\prod_{i=1}^{s_{v}} \frac{d \zeta_{i}^{\prime}}{\zeta_{i}^{\prime 2}}\right) \log \sum_{I \subset\left\{1, \ldots, s_{v}\right\}} \prod_{i \in I} \zeta_{i}^{\prime} g^{-\frac{\alpha^{2}}{4 \pi} Q_{v_{I}}^{2}},
$$

and we choose integration contours with $\left|\zeta_{i}^{\prime}\right|=\varepsilon / s_{v}$ to get

$$
\left|F^{*}(v)\right| \leqq\left(\frac{s_{v}}{\varepsilon}\right)^{s_{v}} g^{\frac{\alpha^{2}}{4 \pi} \sum_{j=1}^{s_{v}} Q_{v_{j}}^{2}}\left|\log \left(1-\left(1+\frac{\varepsilon}{S_{v}}\right)^{s_{v}}+1\right)\right| \leqq B S_{v} ! D_{1}^{s_{v}} g^{\frac{\alpha^{2}}{4 \pi} \sum_{i=1}^{s_{v}} Q_{v_{v}}^{2}}
$$

where $B$ and $D_{1}$ are suitable constants. This will be considered together with (3.24) and

$$
n R=\sum_{v>r, v \text { n.t. }} n_{v}\left(h_{v}-h_{v^{\prime}}\right)+\sum_{\text {endpoints } i}\left(R-h_{i}\right)-n,
$$

where $v^{\prime}$ is again the non-trivial vertex preceding immediately $v$ in $\gamma,\left(v^{\prime}=r\right.$ and $h_{v^{\prime}}=-1$ if no such vertex exists) and $h_{i}$ is the frequency label of the first non-trivial vertex of $\gamma$ to which the $i$-th endpoint is attached.

The (3.25), (3.29) yield the bound, $\left(a \equiv \frac{\alpha^{2}}{4 \pi}-2\right)$ :

$$
\begin{aligned}
\left|\mu_{n}\right| \leqq & \mathscr{N} g^{-2 R-n a R+2(n-1)-\frac{\alpha^{2}}{4 \pi} n} \\
& \left.\times \sup _{\theta, \underline{\sigma} \sum_{\theta}} \sum_{v \text { n.t. }} \prod^{-\frac{\alpha^{2}}{4 \pi} Q_{v}^{2}\left(h_{v}-h_{v^{\prime}}-1\right)} g^{\frac{\alpha^{2}}{4 \pi} n_{v}\left(h_{v}-h_{v^{\prime}}\right)} g^{-2\left(n_{v}-1\right)\left(h_{v}-h_{v^{\prime}}\right)} B D_{1}^{S_{v}}\right]
\end{aligned}
$$

where $\mathscr{N}$ is the number of trees with $n$ distinguished endpoints without counting the different frequency labels and $\sum_{\underline{h}_{\theta}}^{*}$ is the summation over the ways of putting frequency labels $\underline{h}_{\theta}$ on a "tree shape" (unlabeled tree $\gamma$ ), i.e. $\sum_{\underline{h}_{\theta}}^{*}$ is the sum over all the tree shapes $\theta$ of a tree $\gamma$ such that $Q_{v} \neq 0$ if $v>v_{0}, v_{0}$ being the first non-trivial vertex of $\gamma$, and $Q_{v_{0}}=0$.

A simple counting argument gives

$$
\mathscr{N} \leqq 2^{4 n}, \quad \sum_{v \text { n.t. }} s_{v} \leqq 2(n-1)
$$

so that, using (3.30)

$$
\begin{aligned}
\left|\mu_{n}\right| \leqq & D_{2}^{n} \sup _{\theta, \underline{\underline{h}}} \sum_{\underline{h} \theta} *\left(\prod_{i=1}^{n} g^{-a\left(R-h_{i}\right)}\right) g^{-2\left(R-h_{v_{0}}\right)} \prod_{v>v_{0}, v \text { n.t. }} g^{-a\left(h_{v}-h_{v^{\prime}}\right)} \\
& \leqq D_{2}^{n}\left(1-g^{-2}\right)^{-1}\left(1-g^{-a}\right)^{-n}
\end{aligned}
$$

for a suitable constant $D_{2}$. This proves that the Mayer expansion is uniformly convergent in $\Lambda$ with radius of convergence bounded below by $1 /\left(D_{2}\left(1-g^{-a}\right)^{-1}\right)$.

Making use of the extra factor $g^{-\frac{\alpha^{2}}{4 \pi} Q_{v_{0}}^{2} h_{v_{0}}}$ present when $Q_{v_{0}} \neq 0$, one can also show that the "non-neutral trees" give a vanishing contribution to $\mu_{n}$ as $R \rightarrow \infty$. 


\section{The Correlation Functions}

We can use (2.12) to find an explicit expression for the truncated correlation functions in terms of the kernels $\bar{V}(\gamma, \underline{\sigma}, \underline{x})$. One can in fact think that the expression in the exponent of (2.12) is simply $\left(a=\frac{\alpha^{2}}{4 \pi}-2\right)$ :

$$
\sum_{\sigma= \pm 1} \frac{\lambda}{2} g^{-a R} \int_{\Lambda g^{-R}}\left[1+\sum_{j=1}^{k} g^{-2 R} \delta\left(x-g^{-R} x_{j}\right) \delta_{\sigma \sigma_{j}} \omega_{j}\right]: e^{i \sigma \alpha \phi_{x}^{(<R)}}: d x,
$$

so that we see that the calculation of the integral (2.12) can be performed via (3.22) replacing $\lambda$ by $\lambda\left[1+\sum_{j=1}^{k} g^{-2 R} \delta\left(x-g^{-R} x_{j}\right) \delta_{\sigma \sigma_{j}} \omega_{j}\right]$ and retaining only the terms of first order in $\omega_{1}, \ldots, \omega_{k}$. We find

$$
\begin{aligned}
\varrho_{\Lambda}^{T}(\underline{x}, \underline{\sigma})= & \sum_{n \geqq k} \sum_{\gamma, h_{r}=-1}^{(n, \underline{x}, \underline{\sigma})} g^{-2 R k}\left(\frac{\lambda}{2} g^{-\frac{\alpha^{2}}{4 \pi}-a R}\right)^{n} \\
& \times \int_{\left(g^{-R} \Lambda\right)^{n-k}} \frac{d y_{1} \ldots d y_{n-k}}{\prod_{v} s_{v} !} \prod_{v \text { n.t. }} F(v) g^{\left(-\frac{\alpha^{2}}{4 \pi} Q_{v}^{2}+\frac{\alpha^{2}}{4 \pi} n_{v}\right)\left(h_{v}-h_{v^{\prime}}\right)},
\end{aligned}
$$

where $\sum^{(n, x, g)}$ means that the sum is over the trees with $n$ endpoints, between which there are the "frozen vertices" $x_{1}, \ldots, x_{k}$ with charges $\sigma_{1}, \ldots, \sigma_{k} ; y_{1}, \ldots, y_{n-k}$ are the positions of the other endpoints.

The fact that in (4.2) there are less integrations (and less $g^{-2 h_{v}}$ factors) than in (2.12) is overcompensated by the factor $g^{-2 R k}$, so that, up to a $k !\left(\begin{array}{l}n \\ k\end{array}\right)$ factor, one can do the same estimate as in Sect. 3, thus proving the convergence of the Mayer expansion for the truncated correlation functions.

In order to study the decay properties of the correlations, one needs a more accurate bound, which takes exactly into account the missing $g^{-2 h_{v}}$ factors and the constraints related to the positions of the frozen vertices. Given a tree $\gamma$, we define its "skeleton" $\tilde{\gamma}$ as the tree which is obtained from $\gamma$ by eliminating all the branches which are not needed to connect the frozen vertices to the root. Proceeding as in Sect. 3, one can show that the contribution of $\gamma$ to $\varrho^{T}(\underline{x}, \underline{\sigma})$ is bounded by $\left(h_{v} \equiv R\right.$ if $v$ is an endpoint)

$$
(c \lambda)^{n} \sum_{\left\{h_{v}\right\} \text { v.t. }}^{*} g^{-2\left(R-h_{v_{0}}\right)}\left(\prod_{\substack{v>v_{0} \\ v \text { n.t. or endpoint }}}^{\dot{m}} g^{-a\left(h_{v}-h_{v^{\prime}}\right)}\right)\left(\prod_{\tilde{\gamma} \ni v \text { n.t. }} g^{-2\left(R-h_{v}\right)\left(\tilde{s}_{v}-1\right)}\right),
$$

where $\tilde{s}_{v}$ is the number of vertices following immediately $v$ in $\tilde{\gamma}, c$ is a suitable constant and $\Sigma^{*}$ means that there is, for any $v \in \tilde{\gamma}$, the constraint

$$
h_{v} \leqq \bar{h}_{v}(\underline{x})=\max \left\{h: \exists \Delta \in Q^{h} \text { such that } g^{-R} x_{i} \in \Delta, \forall x_{i} \in \underline{x}(v)\right\} .
$$

In order to bound (4.3), we need another definition. Let $\tilde{\gamma}$, the "superskeleton" of $\gamma$, be the tree which is obtained by adding to the skeleton $\tilde{\gamma}$ some lines of $\gamma$, starting in a non-trivial vertex $v \in \tilde{\gamma}$ or in $v_{0}$ and ending in an endpoint, so that, for any $v \in \tilde{\gamma}$, the number $\hat{s}$ of vertices following immediately it in $\hat{\gamma}$ is equal to $s_{v}$. 


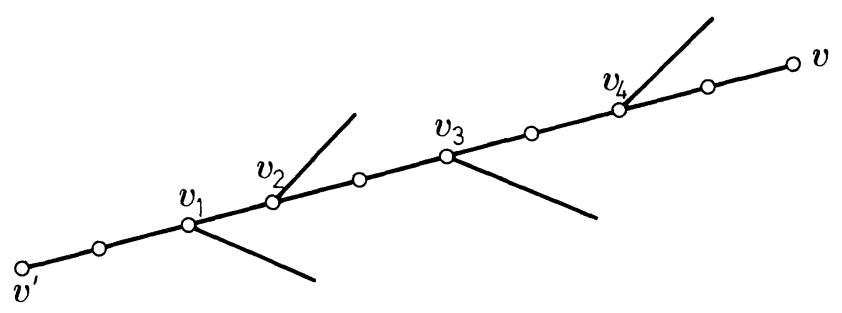

Fig. 2

Let $L_{v^{\prime} v}$ be a line of the superskeleton $\hat{\gamma}$ joining the vertex $v^{\prime}$ and the vertex $v$ immediately following it (in $\hat{\gamma}$ ). Such line, thought of as a line in $\gamma$, contains other non-trivial vertices $v_{1}<v_{2}<\ldots<v_{r}$.

It is easy to see that we can decompose the expression (4.3) so that to the line $L_{v^{\prime} v}$ is associated the factor

$$
\begin{aligned}
& S_{v^{\prime} v}=g^{-a\left(h_{v}-h_{v_{r}}\right)} \prod_{i=1}^{r}\left[\sum_{\substack{\left\{h_{\tilde{\nu}}\right\} \tilde{v} \geq v_{i} \\
\tilde{v} \text { n.t. }}}\left(\prod_{\substack{\tilde{v} \geq v_{i} \\
v \\
v \text { n.t. or endpoint }}} g^{-a\left(h_{\tilde{v}}-h_{\tilde{v}}\right)}\right)\right]
\end{aligned}
$$

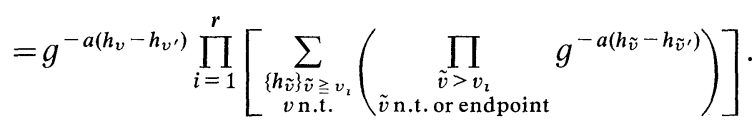

$S_{v^{\prime} v}$ is easily estimated by noticing that to each non-trivial vertex $\tilde{v} \geqq v_{i}, i=1, \ldots, r$, one can associate (in a non-unique way) a line starting in $\tilde{v}$ and ending in an endpoint and that one can collect the $g^{-a\left(h_{\tilde{v}}-h_{\tilde{v}}\right)}$ factors so that each line carries a factor $g^{-a\left(R-h_{\hat{v}}\right)}$. Then

$$
S_{v^{\prime} v} \leqq g^{-a\left(h_{v}-h_{v^{\prime}}\right)} c^{\left(\sum_{i=1}^{r} n_{v_{\mathrm{v}}}\right)}
$$

and (4.3) can be bounded by

$$
(c \lambda)^{n} \sum_{\left\{h_{v}\right\}_{v \in \hat{\gamma}} \text { n.t. }}^{*} g^{-2\left(R-h_{v_{0}}\right)}\left(\prod_{\begin{array}{c}
v \in \hat{\gamma}, v>v_{0} \\
v \text { n.t. or end point }
\end{array}} g^{-a\left(h_{v}-h_{\tilde{v}^{\prime}}\right)}\right)\left(\prod_{\tilde{\gamma} \ni v \text { n.t. }} g^{-2\left(R-h_{v}\right)\left(\tilde{s}_{v}-1\right)}\right),
$$

where $\tilde{v}^{\prime}$ is the vertex immediately preceding $v$ in $\hat{\gamma}$.

The bound (4.7) implies very easily that in the expansion (4.2), if $\lambda$ is small enough, the leading terms are those with the minimum number, say $n_{0}$, of endpoints sufficient to satisfy the neutrality constraint $Q_{v_{0}}=0$. Of course

$$
n_{0}=k+\left|\sum_{i=1}^{k} \sigma_{i}\right| \text {. }
$$

If $k=2$ and $\sigma_{1}+\sigma_{2}=0$, there is only one leading tree and one can easily show, using (4.4), that

$$
\lim _{\tilde{d}\left(x_{1}, x_{2}\right) \rightarrow \infty} \tilde{d}\left(x_{1}, x_{2}\right)^{\frac{\alpha^{2}}{2 \pi}} \varrho^{T}\left(x_{1}, x_{2} ; \sigma_{1}, \sigma_{2}\right)
$$

is different from zero and finite, if the hierarchical distance $\tilde{d}\left(x_{1}, x_{2}\right)$ is defined as in (2.10). 
For increasing values of $k$, one could obtain similar results with increasing work. In any case (4.7) implies immediately at least the upper bound

$$
\left|\varrho^{T}(\underline{x}, \underline{\sigma})\right| \leqq c|\lambda|^{n_{0}} \tilde{d}(\underline{x})^{-\frac{\alpha^{2}}{4 \pi} k}
$$

where $\tilde{d}(\underline{x})=\min \left\{\tilde{d}\left(x_{i}, x_{j}\right), i \neq j\right\}$.

\section{References}

1. Benfatto, G., Cassandro, M., Gallavotti, G., Nicolò, F., Olivieri, O., Presutti, E., Scacciatelli, E.: Some probabilistic techniques in field theory. Commun. Math. Phys. 59, 143 (1978)

2. Benfatto, G., Cassandro, M., Gallavotti, G., Nicolò, F., Olivieri, O., Presutti, E., Scacciatelli, E.: Ultraviolet stability in Euclidean scalar field theories. Commun. Math. Phys. 71, 95 (1980)

3. Benfatto, G., Gallavotti, G., Nicolò, F.: On the massive sine-Gordon equation in the first few regions of collapse. Commun. Math. Phys. 83, 387 (1982);

Nicolò, F.: On the massive sine-Gordon equation in the higher regions of collapse. Commun. Math. Phys. 88, 581 (1983)

4. Fröhlich, J., Spencer, T.: The Kosterlitz-Thouless transition in two dimension Abelian spin systems and the Coulomb gas. Commun. Math. Phys. 81, 527 (1981)

5. Gallavotti, G., Nicolò, F.: The screening phase transitions in the two dimensional Coulomb gas. J. Stat. Phys. 39, 133 (1985)

6. Nicolò, F., Renn, J., Steinmann, A.: The pressure of the two dimensional Coulomb gas at low and intermediate temperatures. Ann. Inst. H. Poincaré (to appear)

7. Nicolò, F., Renn, J., Steinmann, A.: The sine-Gordon equation in all regions of collapse. Commun. Math. Phys. (in press)

8. Benfatto, G.: An iterated Mayer expansion for the Yukawa gas. J. Stat. Phys. (to appear)

9. Speer, E.R.: Mayer coefficients in two dimensional Coulomb systems. J. Stat. Phys. (to appear)

10. Gallavotti, G.: Renormalization theory and ultraviolet stability for scalar fields via renormalization group methods. Rev. Mod. Phys. 57, 471 (1985)

11. Wilson, K., Kogut, J.: The renormalization group and the $\varepsilon$-expansion. Phys. Rep. 12, 75 (1974)

Communicated by J. Fröhlich

Received January 1, 1986 